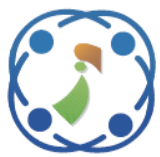

\title{
Plus-Shape of Mushroom-Like EBG with Square Microstrip Emitter to Expand the Working Space in Dielectric Heating Applications
}

\author{
Nuchanart Santalunai ${ }^{1} \quad$ Samran Santalunai $^{2 *}$ \\ Piyaporn Meesawad ${ }^{3}$ \\ Chanchai Tongsopa $^{2} \quad$ Samroeng Santalunai $^{2}$ \\ ${ }^{I}$ Department of Telecommunication Engineering, Faculty of Engineering and Architecture, \\ Rajamangala University of Technology Isan, Nakhon Ratchasima 30000, Thailand \\ ${ }^{2}$ School of Electronic Engineering, Suranaree University of Technology, Nakhon Ratchasima 30000, Thailand \\ ${ }^{3}$ School of Telecommunication Engineering, \\ Suranaree University of Technology, Nakhon Ratchasima 30000, Thailand. \\ * Corresponding author's Email: samran.sa@ sut.ac.th
}

\begin{abstract}
This research focusses on the methods for expanding the working space to radiate the electromagnetic fields of $2.45 \mathrm{GHz}$ within a dielectric load by using only one square patch radiator together with a plus-like electromagnetic band gap (EBG). One port of square patch generates a high transmission power to distribute the symmetrically electromagnetic field distribution. Electromagnetic band gap structure has a unique band gap characteristic where it can suppress the surface wave and continuously increase electric fields distribution on dielectric load under test with dimension of $300 \times 300 \mathrm{~mm}^{2}$ when the thickness dimension is optimized. A dielectric heating applicator using microstrip patch with EBG structure has a low-loss dielectric because of the structure of system which has no gap between applicator surface and dielectric load. This paper provides a comparison of the simulation of electric distribution in microwave chamber when the applicators are copper plate, square patch microstrip and square patch generator surrounded by EBG. In the simulation results, an electric distribution in the dielectric load was compared when the dielectric constant was 4.7. The maximum percentage of electric fields distribution that was evenly distributed in dielectric load was about $99.97 \%$ at middle surface with a thickness of $5 \mathrm{~mm}$ by using square patch generator surrounded by EBG as the applicator. In addition, the simulation results were confirmed with the results of the dielectric heating measurement of the dielectric load as rice which is a popular grain in the commercial application of dielectric industrial drying.
\end{abstract}

Keywords: Electromagnetic band gap, Dielectric heating, Rice, Electric field distribution, Applicator.

\section{Introduction}

The thermal phenomena of using the profit of electromagnetic waves is an important topic in scientific and engineering research [1-3]. At present, various types of heating processes have been developed, such as induction heating and dielectric heating. Induction heating or magnetic field heating is the process of heating an electrically conducting object of metallic material by inducting a magnetic field to carry an electric current through it, especially with steel objects. Most current flows through objects at the level of skin depth, this causes a current to flow around the work piece until it is heated to the surface of the work piece. The thermal process occurring depends on the volume of current being induced and the equivalent resistance along the path through which the current passes, and then transferred to other areas by conduction and convection. Moreover, another electromagnetic field application is dielectric heating or electric field heating, this process is used to heat non-metal materials $[4,5]$. Dielectric heating is a process that can directly transfer heat into a dielectric material that is suitable for the resonant frequency without affecting other materials, owing to the heating that occurs when dielectric molecules vibrate and cause friction between the polar 
molecules to generate heat $[6,7]$. Dielectric heating is applied on the principle of frequency conductivity in the form of an electric field by transmitting power to the dielectric medium based on Eq. (1) [8],

$$
P=E^{2} \sigma=2 \pi f E^{2} \varepsilon_{o} \varepsilon^{\prime \prime}
$$

Where $E$ is the rms of electric field strength on the material $(\mathrm{V} / \mathrm{m}), P$ is the power density $\left(\mathrm{W} / \mathrm{m}^{3}\right)$, $\sigma$ is conductivity, $f$ is frequency ( $\mathrm{Hz}), \varepsilon_{o}$ is permittivity of free space $(F / m), \varepsilon^{\prime \prime}$ is loss factor of material. The changing rate of the temperature increased, $\Delta T / \Delta t$ in ${ }^{\circ} \mathrm{C} / \mathrm{s}$, in the dielectric material caused by the conversion of energy from the electric field to heat in the material as Eq. (2). Where, $c_{p}$ is specified heat of the material $\left(\mathrm{kJ} / \mathrm{kg}^{\circ} \mathrm{C}\right), \rho$ is density of the material $\left(\mathrm{k} / \mathrm{m}^{3}\right)$

$$
\frac{\Delta T}{\Delta t}=\frac{P}{\rho c_{p}}
$$

Dielectric heating is classified into two operations in the high frequency range: a radio frequency (RF) alternating electric field heating and microwave electromagnetic radiation heat dielectric material $[9,10]$. The radio wave heating process is caused by an electric field between the electrode plates, which is a line of electric force in the radio frequency band from $27 \mathrm{MHz}$ to $40 \mathrm{MHz}$ [11]. In terms of microwave heating, the frequency is used at $915 \mathrm{MHz}$ or $2.45 \mathrm{GHz}$. The principle of operation of microwave heating is transverse wave in waveguide from one place to another. Each resonant frequency has different ability to penetrate into materials [12]. Radio frequency and microwave dielectric heating technology, both electromagnetic heating processes, have more applications in agriculture such as in the drying industry, fruit preservation industry, wood drying [13-16]. In addition, dielectric heating can also be applied to living organisms or pests in agriculture, such as the control of rice weevils or worms that are embedded or hidden within agricultural products or seeds and plants [17-19]. The type of the frequency band to use depends on the properties of the electromagnetic or material that requires heat. Radio frequency heating is suitable for materials that are combined and have distinct dielectric loss values. Microwave heating is distinct from RF heating in that an electromagnetic wave is launched from small dimension emitter at high frequency with shorter wavelength. This wavelength is suitable for materials containing water molecules or polar molecules. This will absorb the energy of the

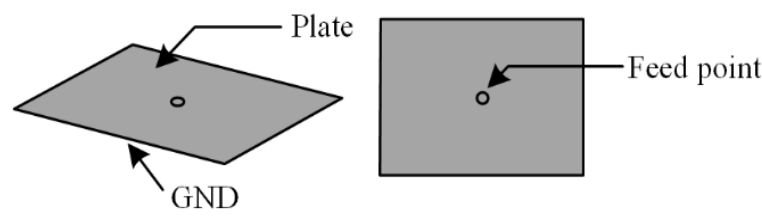

(a)

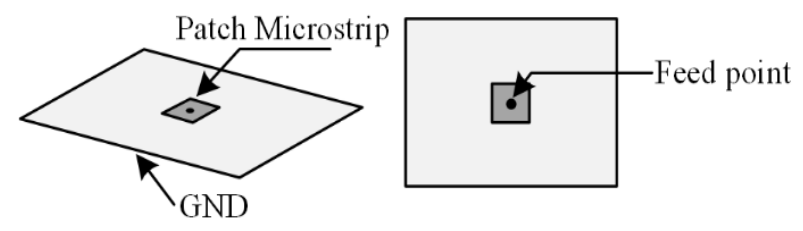

(b)

Figure. 1 Structure of: (a) plate emitter in RF heating and (b) microstrip patch emitter in MW heating

waves that pass through it and generate heat [20]. Fig. 1 shows the difference in the dimension of the emitter at RF and MW frequencies. The emitter of MW heating is smaller than the one in RF heating, so the area to radiate electric distribution or heating power is less followed. This is the reason why microwaves are not used in large industries; the application area is too small due to wavelength limitation. Even so, MW heating has the ability to heat very well. Therefore, it can be said that if the efficiency of the microwave emitter can be enhanced into a more usable area, it will be very good for the dielectric heating technology.

Periodic structures are suitable for expanding the working space of MW heating application. There are abundant in nature and have several characteristics such as frequency stop band, pass band, and band gaps [21-23]. The domain of the applications is seen in filter designs, grating, frequency selective surfaces (FSS), photonic crystals and photonic band gap (PBG). They are classified under definition of Electromagnetic Band Gap (EBG) structure [24]. The novel shapes of mushroom-like EBG structures were considered in our previous paper [25], unique properties have made them applicable in many antenna and microwave application. EBG structures are periodic structures which can suppress the propagation of electromagnetic waves in particular frequency bands, increase in-phase reflection coefficient bandwidth and distribute electromagnetic field based on the dimensional periodicity of EBG structure. They are categorized into many shapes according to resonant bandwidth such as square shape, plus shape, and cross shape. Thus, the remainder of the paper concentrating on expanding the working space of MW dielectric heating by using a square microstrip emitter together with EBG 
surface. A microstrip emitter performs as an external source which activates the EBG performance.

The rest of this article is organized as follows: Section 2 presents the designed model and simulation results of plus shape EBG and designed applicator. Section 3 describes electric field distribution at dielectric load analysis. The performances of square patch around by plus shaped EBG applicator is validated to investigate the simulated results in 4 . Section 5 summarizes the paper and describes future work.

\section{Designed model and simulation results of plus shape EBG unit cell and applicator}

In this section, a plus shaped EBG was simulated to exhibit the performance of reflection phase and band gap. Both incident plane wave and suspended microstrip line approach were utilized to investigate the performance of this shape. Moreover, a square patch microstrip with input impedance of $13.8 \mathrm{ohms}$ was designed to perform ability to radiate electric field distribution of an emitter compared with a copper plate and a square patch microstrip with the EBG.

\subsection{Plus shape EBG configurations}

The parameters of the EBG structure are labeled in Fig. 2 and Table 1. The configuration consisted of three parts that are: plus shaped patches, polypropylene substrate and ground plane. The conducting patches were connected to ground plane with small pin, called vias. One element of the EBG is that it looked like mushroom whereby the patches array was seen to be the unit cell by considering vias by vias, which is smaller than the wavelength at 2.45 GHz. The operation of the EBG unit cell is performed based on lumped-circuit (LC) elements as illustrated in Fig. 3. The LC model introduced an inductor (L) and capacitor (C), which were due to the current flowing through the vias and the gap between adjacent patches, respectively. The impedance of resonance circuit can be approximated by the following formulas [24]:

$$
Z=\frac{j \omega L}{1-\omega^{2} L C}
$$

The resonance frequency of the circuit is given by:

$$
\omega_{o}=\frac{1}{\sqrt{L C}}
$$

The value of the capacitor can be proven using a parallel circuit calculation technique between
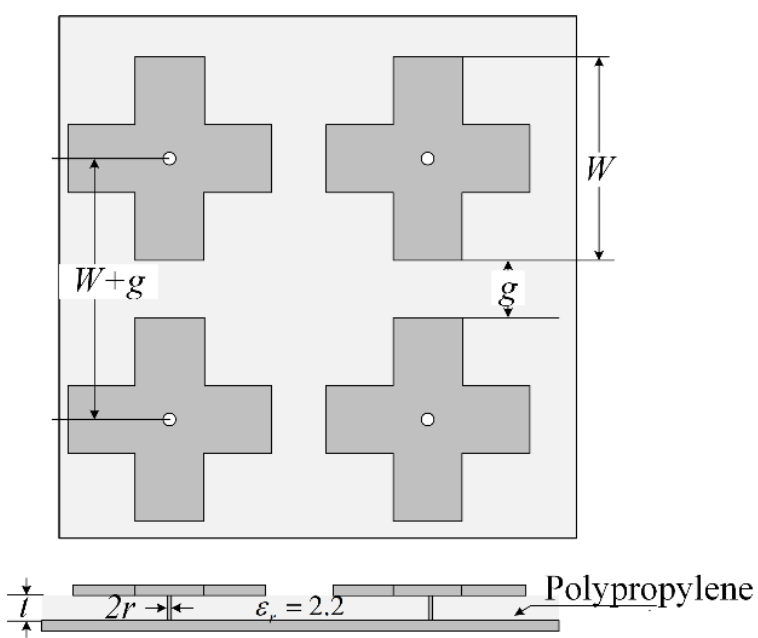

Figure. 2 Unit cell of plus shaped EBG
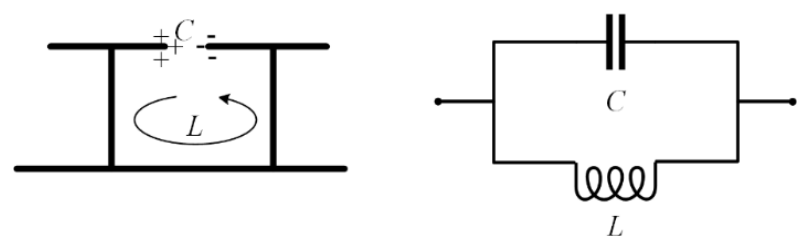

Figure. $3 \mathrm{LC}$ model for plus shaped EBG structure

capacitor and inductor as shown in the following equation:

$$
C=\frac{W \varepsilon_{o}\left(1+\varepsilon_{r}\right)}{\pi} \cos ^{-1}\left(\frac{2 W+g}{g}\right)
$$

The value of the inductor arose from the current that flowed through the vias and patch in Fig. 2 (b):

$$
L=\mu t
$$

where, $\mu$ is permeability and $\varepsilon$ is permittivity.

The interesting EBG characteristics are not reflected the in-transmission wave and suppressed the surface wave in the resonance band gap. Fig. 4 (a) depicts a model for plus shaped EBG analysis based on periodic boundary condition [26]. When the incident wave is a plane wave in CST software that is traveling to the EBG surface for the distance of half wavelength 4 (b), the reflection phase of the EBG structure varies with frequency as plotted in Fig. 5 (a). At a certain frequency, the reflection phase is zero degrees which does not exist in perfect electric conductor reflector plane. Additionally, the microstrip line with two port sets over the surface of EBG unit cell as shown in Fig. 4 (c) [27]. The frequency band gap characteristic through which the surface wave cannot propagate is illustrated in Fig. 5 (b). Only transmission coefficient $S_{21}$ is presented for lucidity at frequency range with $S_{21}$ below $-20 \mathrm{~dB}$ extend from 2.3 to $2.55 \mathrm{GHz}$. 


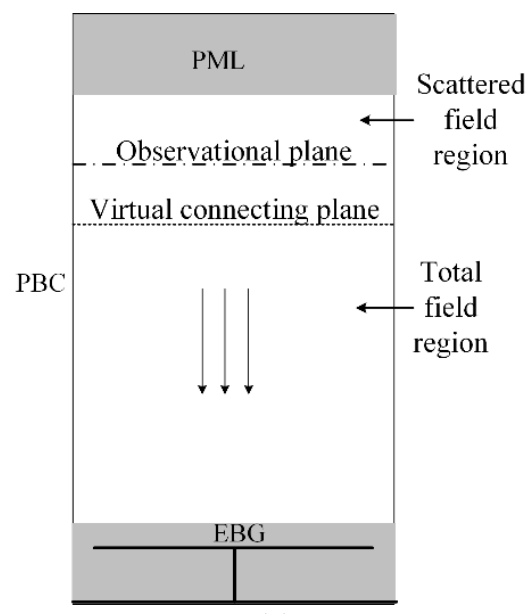

(a)

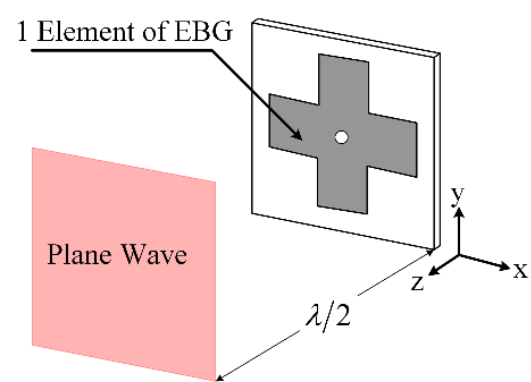

(b)

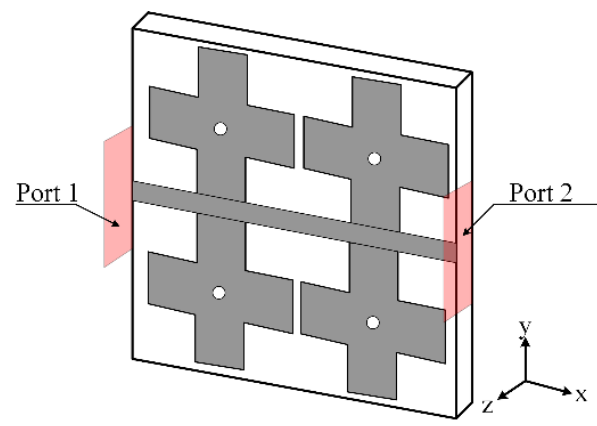

(c)

Figure. 4 Sketch of simulated EBG characteristics: (a) periodic boundary condition, (b) 1 element of EBG, and (c) plus liked EBG unit cell with suspended microstrip structure

\subsection{Square patch microstrip emitter}

An emitter of dielectric heating system should have a symmetrical and low-profile structure because the EBG elements can be arranged in an orderly manner. A microstrip antenna or printed antenna that consists of three layers, same as the proposed EBG, is mostly used at microwave frequencies. Therefore, this research designed a square patch microstrip at 2.45 $\mathrm{GHz}$ for utilizing emitter of applicator in dielectric heating system as shown in Fig. 6, based on patch microstrip antenna theory [28]. Input impedance of patch emitter is dependent on the magnetron tube impedance that is $13.8 \mathrm{ohms}$ at $2.45 \mathrm{GHz}$. Due to the
Table 1. The parameters of plus shaped EBG

\begin{tabular}{|l|l|}
\hline Parameters & Size $(\mathbf{m m})$ \\
\hline Patch width $(W)$ & 43.75 \\
\hline Gap width $(g)$ & 1.012 \\
\hline Substrate thickness $t()$ & 2.00 \\
\hline Dielectric constant $\left(\varepsilon_{r}\right)$ & 2.20 \\
\hline Radius of vias $(r)$ & 2.00 \\
\hline Width of unit cell $(W+g)$ & 44.50 \\
\hline
\end{tabular}

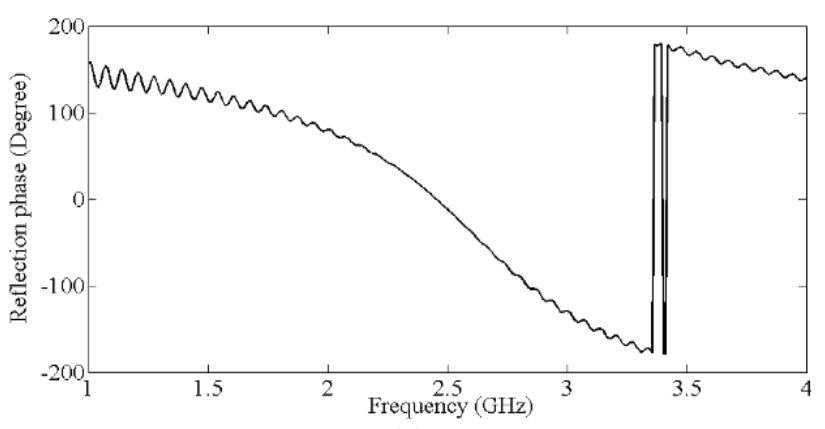

(a)

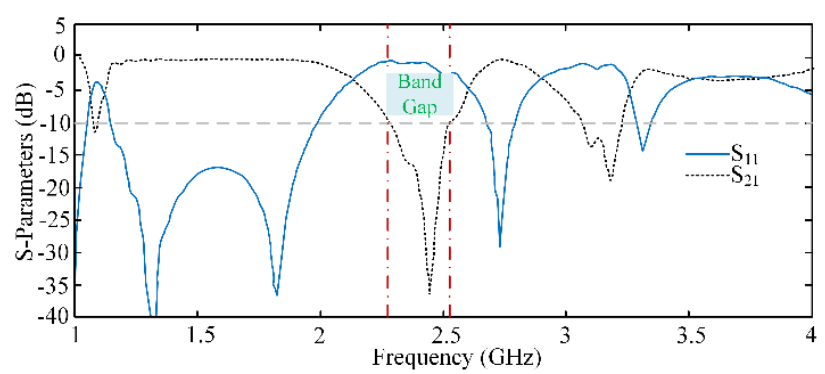

(b)

Figure .5 Simulated results of EBG characteristics: (a) reflection phase of 1 element plus shaped EBG and (b) SParameter of suspended microstrip on plus shaped EBG unit cell

Table 2. Parameters of square patch emitter

\begin{tabular}{|l|c|}
\hline \multicolumn{1}{|c|}{ Parameters } & Size $(\mathbf{m m})$ \\
\hline Width of square patch $\left(W_{P}\right)$ & 84.875 \\
\hline Length of square patch $\left(L_{P}\right)$ & 84.875 \\
\hline Width of ground plane $(W)$ & 56.250 \\
\hline Length of ground plane $(L)$ & 56.250 \\
\hline Dielectric constant $\left(\varepsilon_{r}\right)$ & 2.20 \\
\hline Substrate thickness $\left(\varepsilon_{r}\right)$ & 2.00 \\
\hline
\end{tabular}

symmetrical design of the structure, the width and length of the patch were adjusted. The results of the adjustment are shown in Fig. 7. Table 2 compiles the parameters value of the proposed emitter.

\subsection{Comparison of E-fields distribution on various emitter}

In this section, the various electromagnetic emitters of the dielectric heating systems are 

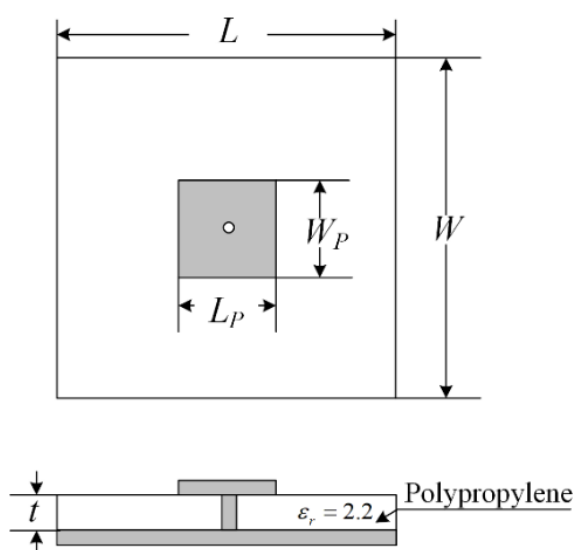

Figure. 6 The configuration of square patch microstrip emitter

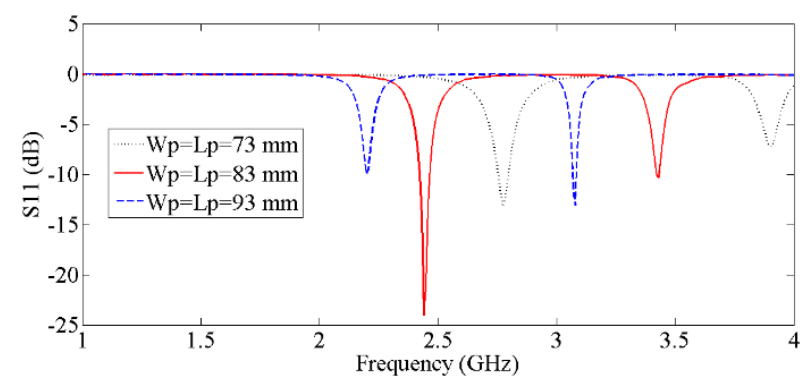

Figure. 7 Simulated S11 of square patch microstrip emitter when width and length are varied

\subsubsection{Plate applicator}

Fig. 8 (a) shows plate emitter components comprising of cooper plates, feed point and ground. The total size of cooper plate is $300 \times 300 \mathrm{~mm}^{2}$. The electric fields distribution on plate applicator surface

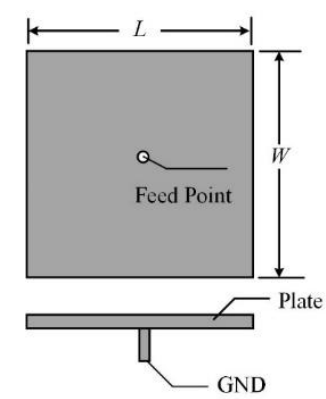

(a)

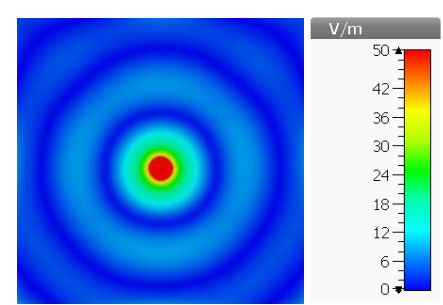

(b)

Figure. 8 A plate applicator: (a) structure and (b) electric field distribution simulation result

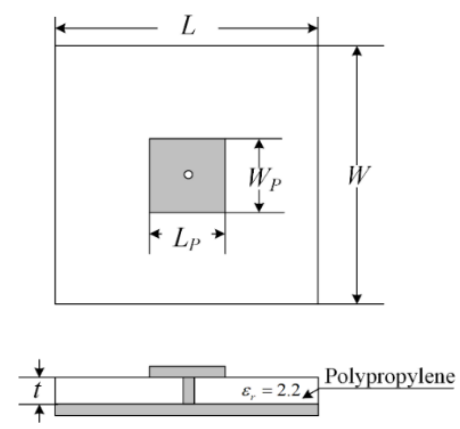

(a)

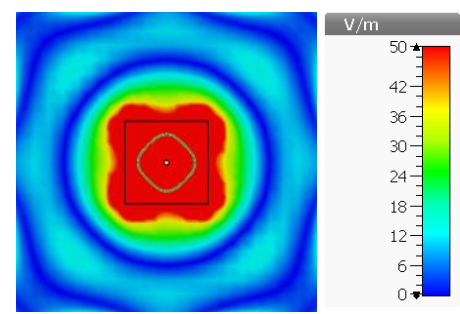

(b)

Figure. 9 A patch microstrip emitter: (a) structure and (b) electric field distribution simulation result

illustrated in Fig. 8 (b) confirms that the heating intensity occurred at the center of emitter and spread out with only a small radius when the maximum electric field intensity was $50 \mathrm{~V} / \mathrm{m}$. If this power distribution type is used, result of electric fields distribution will radiate in very small area of heating. The problem may have to be solved by adding more power sources $[29,30]$.

\subsubsection{Square patch microstrip emitter}

A square patch microstrip was designed to generate electromagnetic fields and waves at frequency of $2.45 \mathrm{GHz}$ by matching the input impedance with magnetron source of $13.8 \mathrm{ohms}$. All dimensions were optimized with structural symmetry conditions to obtain symmetrical electric fields distribution as well. The total sizes were $83 \times 83 \mathrm{~mm}^{2}$ when thickness of substrate was $2 \mathrm{~mm}$. The square patch emitter was located on the center of substrate and ground plane and fed at the middle point of structure, as illustrated in Fig. 9 (a). Due to the matching between the source and the emitter, Fig. 9 (b) shows a wider area of electric near field distribution when the total area size is equal to plate emitter and the maximum electric field intensity is 50 $\mathrm{V} / \mathrm{m}$. Furthermore, the working area was improved by using EBG structure.

\subsubsection{Square patch microstrip emitter surrounded with EBG}

A symmetrical microstrip patch emitter with plus shaped EBG was simulated to analyze the electric 

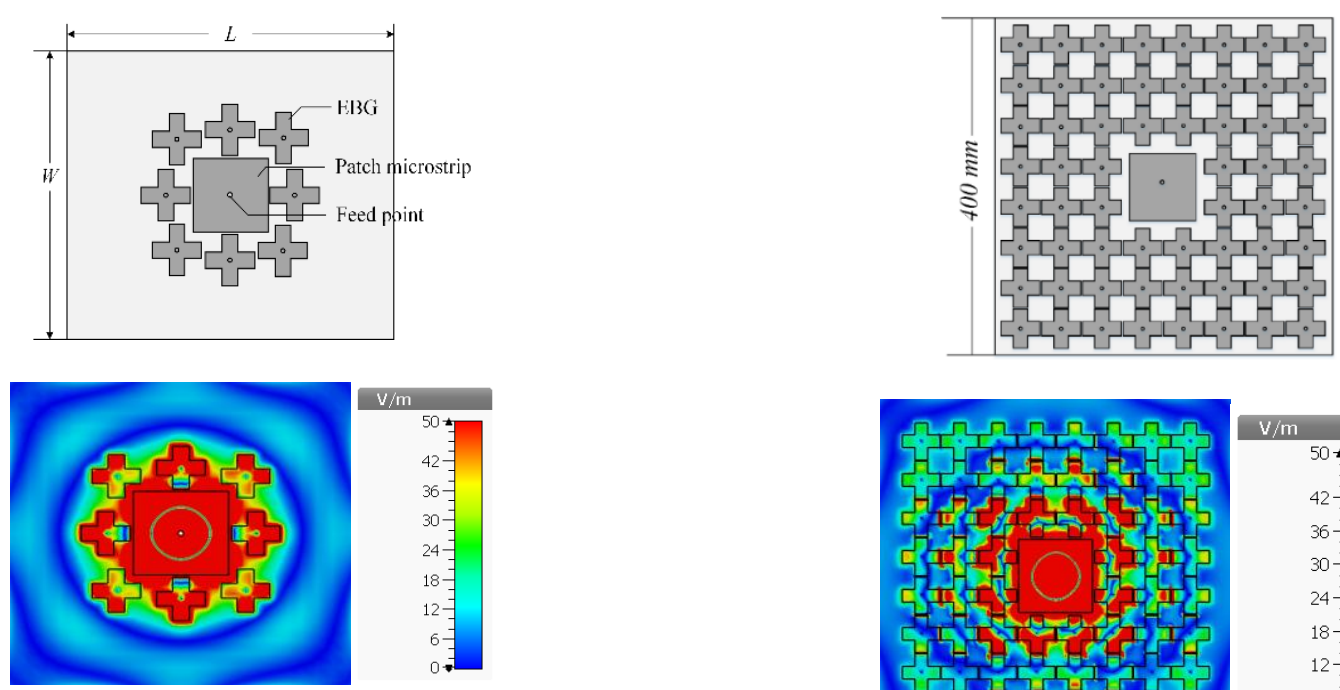

(a)

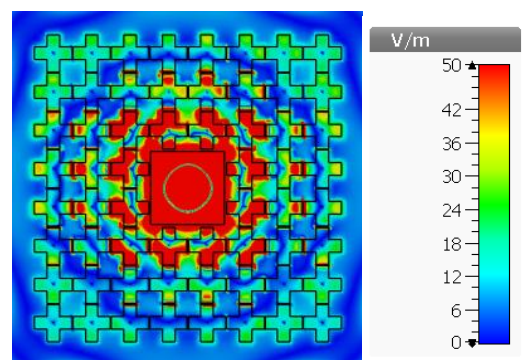

(d)
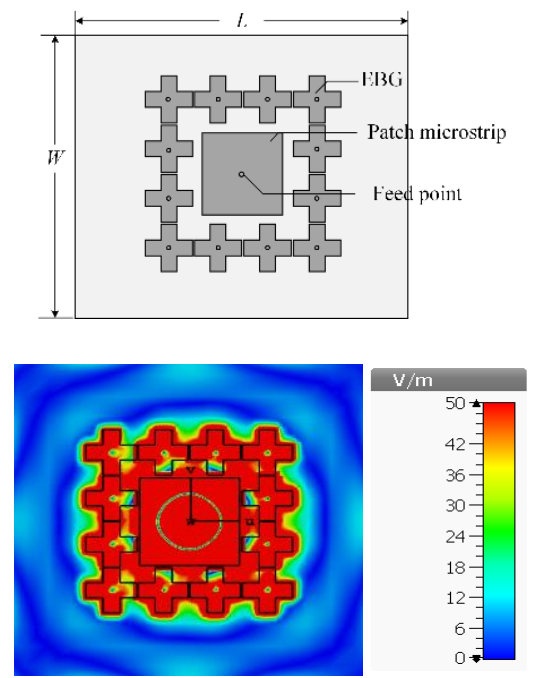

(b)

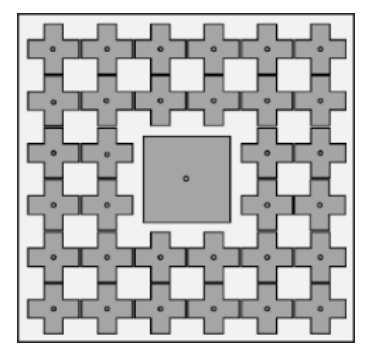

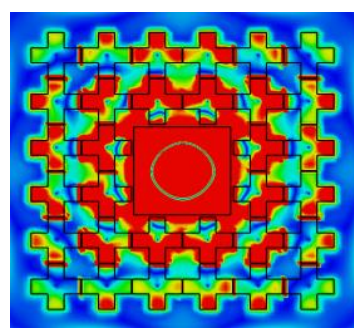

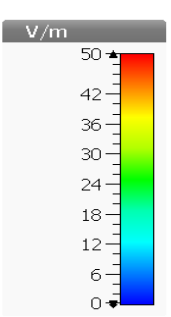

(c)

Figure. 10 A microstrip emitter with variously arrangement of plus shaped EBG and electric field distribution on surface

fields distribution as illustrated in Fig. 10. This started with the analysis of only one round EBG arrangement around the symmetric emitter as shown in Figs. 10 (a) and (b). The second arrangement in Fig. 10 (b) could propagate an electric field in symmetrical square form which is suitable for dielectric load structure. Observing the area of the surrounding electric field, it was found that the electric field could continue to spread further, thus surrounding more of the EBG as denoted in Figs. 10 (c) and (d). The electric field could not be distributed at the edges of structure evenly when the area size was increased to $400 \times 400 \mathrm{~mm}^{2}$, but it could be distributed when the area was $300 \times 300 \mathrm{~mm}^{2}$. Therefore, the arrangement of double round plus liked EBG was used for $2.45 \mathrm{GHz}$ microwave applicator.

\section{Dielectric load and microwave chamber}

The dielectric load in this research was rice with dielectric constant of 4.7. Rice is an important economic crop requiring post-harvest drying process. In terms of dielectric heating simulation, the dielectric load dimension specified the width and length as $300 \times 300 \mathrm{~mm}^{2}$ owing to the results of simulating the working space of the applicator in the previous section although, the parameter $h$ should be analyzed as denoted in Fig. 11 (a). In general, microwave chambers at $2.45 \mathrm{GHz}$ consist of multi sources with rotating table to scatter the microwave into the product [31]. The advantage of the multimode chamber is the even microwave field that enables the heating of large or complex shaped 
Table 3. The view perspective of electric field distribution at darious position on dielectric load

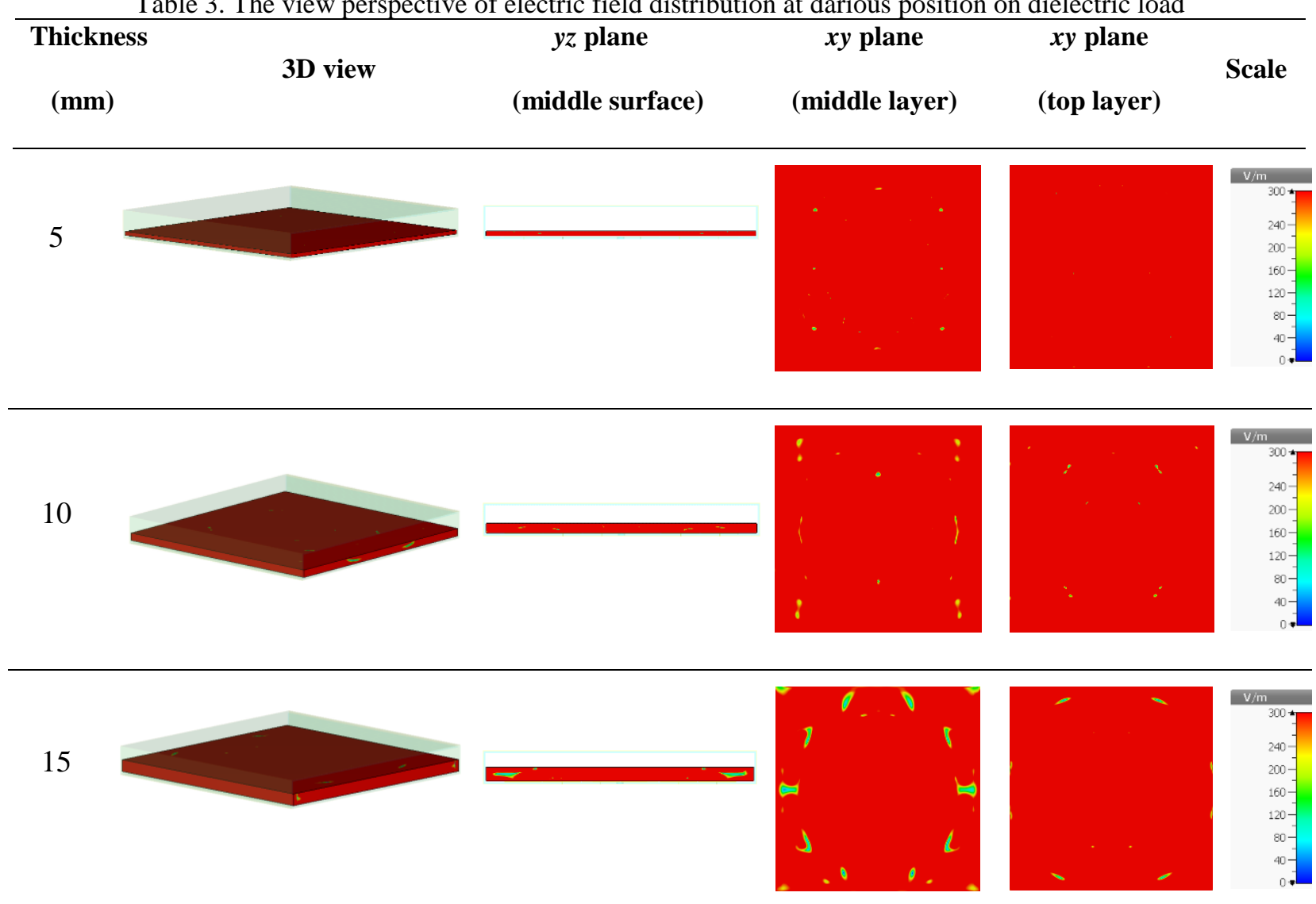
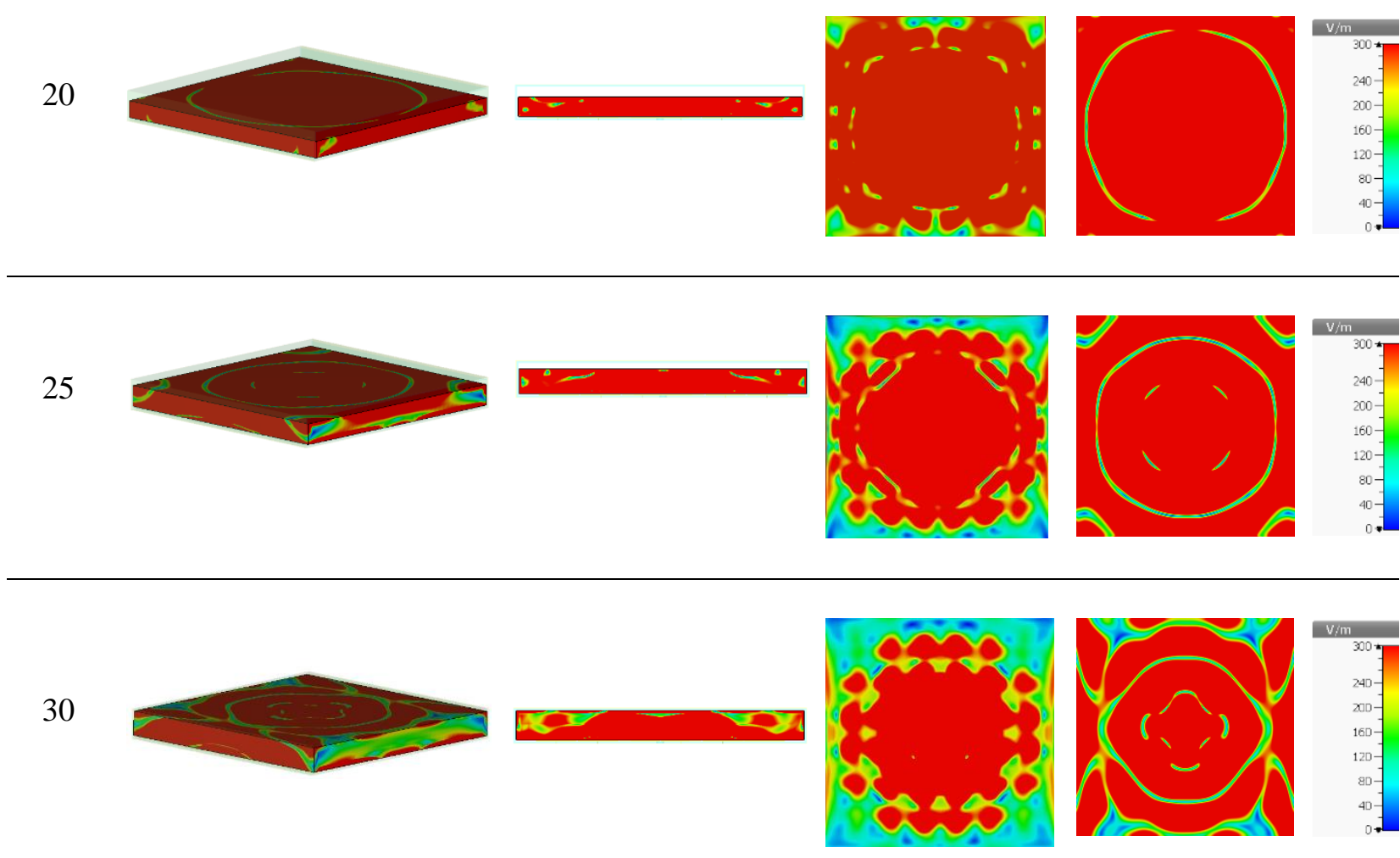


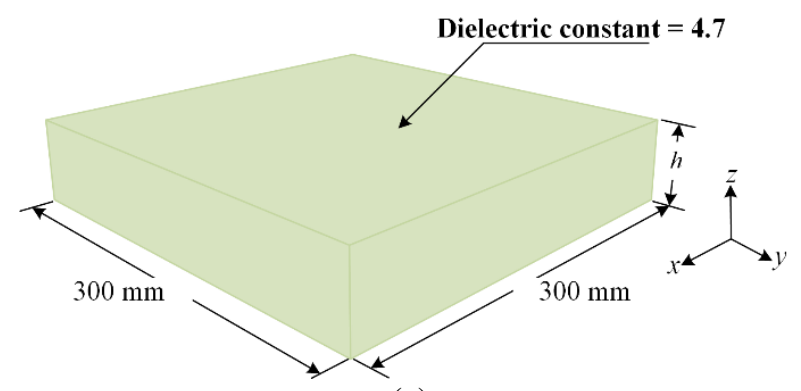

(a)

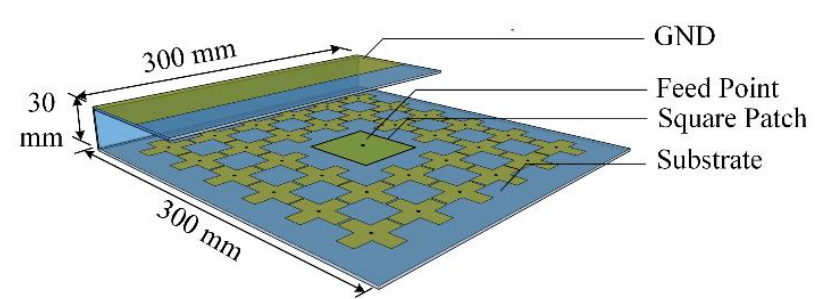

(b)

Figure. 11 Sketch of: (a) a dielectric load structure and (b) proposed applicator

products. However, the distance that microwave field travels from the microwave generator to the product or dielectric load occurring causes the occurrence of a free space propagation loss. Consequently, this research aimed to reduce the losses that occur microwave chamber by using patch microstrip emitter with EBG surface and placing dielectric load close to microwave emitter as shown in Fig. 11 (b). The ground plane was an enclosed microwave chamber at top, bottom, left and right sides. In the simulation setting, the source was set up with input impedance of $13.8 \mathrm{ohms}$.

The simulation results of electric field distribution at the dielectric load in Table 3 show the ability to radiate an electric field to the dielectric load in perspective view, moreover the middle surface of dielectric load in $y z$ plane and $x y$ plane are shown altogether. The parameter $h$ was varied from $5 \mathrm{~mm}$ to $30 \mathrm{~mm}$ by setting the value increments of $5 \mathrm{~mm}$. The electric field distribution effect was uniform and could cover the entire work piece when a dielectric load had a thickness of $5 \mathrm{~mm}$ and $10 \mathrm{~mm}$ with the percentage of electric field distribution of $99.97 \%$ and $98.80 \%$ at middle surface, respectively. However, when the thickness was adjusted to $15 \mathrm{~mm}$ and 20 $\mathrm{mm}$, the electric field was evenly distributed in the middle of the load but a fade of electric field intensity occurred at the edge of the load. As a result, the percentage of electric field distribution was $95.09 \%$ and $83.18 \%$ at middle surface, respectively. It was observed that the electric field intensity diffused slightly at the upper surface of the load than at the central surface of the load due to the nature of the electric field will find the ground plane. Furthermore,
Table 4. Percentage of electric field distribution in dielectric load at $x y$ plane

\begin{tabular}{|c|c|c|}
\hline $\begin{array}{c}\text { Dielectric } \\
\text { thickness }(\mathbf{m m})\end{array}$ & $\begin{array}{c}\text { Middle Layer } \\
(\boldsymbol{\%})\end{array}$ & $\begin{array}{c}\text { Top Layer } \\
(\mathbf{\%})\end{array}$ \\
\hline 5 & 99.97 & 100.00 \\
\hline 10 & 98.80 & 99.73 \\
\hline 15 & 95.09 & 99.27 \\
\hline 20 & 83.18 & 95.01 \\
\hline 25 & 61.37 & 89.52 \\
\hline 30 & 46.33 & 74.15 \\
\hline
\end{tabular}

the electric field intensity disappeared at the edge of the load when the thickness of testing load was 25 $\mathrm{mm}$ and $30 \mathrm{~mm}$. Percentage of electric field distribution calculated from electric field intensity was from $280 \mathrm{v} / \mathrm{m}$ to $300 \mathrm{v} / \mathrm{m}$ as indicated in Table 4.

\section{Microwave performance \\ dielectric heating}

From all the theories and designs including an analysis of the results, this section discusses an applicator fabrication of microwave dielectric heating chamber as shown in Fig. 12. An applicator consists of square patch with center feed point together with second rounds of plus shaped EBG surface. First, the square patch and plus shaped EBG constructed of a copper plate (of $1 \mathrm{~mm}$ thickness) and mounted on a polypropylene (PP) with permittivity of 2.2 was designed to resonate at $2.45 \mathrm{GHz}$. The prototype of microwave dielectric heating machine and dielectric load (rice) with permittivity of 4.7, contained in a polypropylene tray is illustrated in Fig. 13.

The temperature distributed measurement was tested by infrared thermal camera. Rice was placed in a polypropylene tray with the thickness of rice varying from $5 \mathrm{~mm}$ to $30 \mathrm{~mm}$ by setting the value increments of $5 \mathrm{~mm}$. Detail experimental parameters, the power of the proposed microwave dielectric heating used for testing was set at 100 watts and the weight of rice was $450 \mathrm{~g}$ while the dielectric load thickness was $5 \mathrm{~mm}$. The weight was increased according when adding the thickness of the dielectric load. The measurement results are shown in Table 5 . From the testing results, when the maximum and minimum temperatures were controlled at $50{ }^{\circ} \mathrm{C}$ and $28{ }^{\circ} \mathrm{C}$, respectively, it was found that the heating appeared to increase when the heating time was increased for the thickness of dielectric load of $5 \mathrm{~mm}$. Furthermore, when the dielectric load thickness was increased to $10 \mathrm{~mm}$, the heating slowed down over working space at the edge of tray. Increasing dielectric thickness to $15 \mathrm{~mm}, 20 \mathrm{~mm}, 25 \mathrm{~mm}$ and 30 $\mathrm{mm}$, the temperatures mensuration had identical 
Table. 5 Comparison results of dielectric load heating at different thickness over heating time

\section{Dielectric}

Thickness
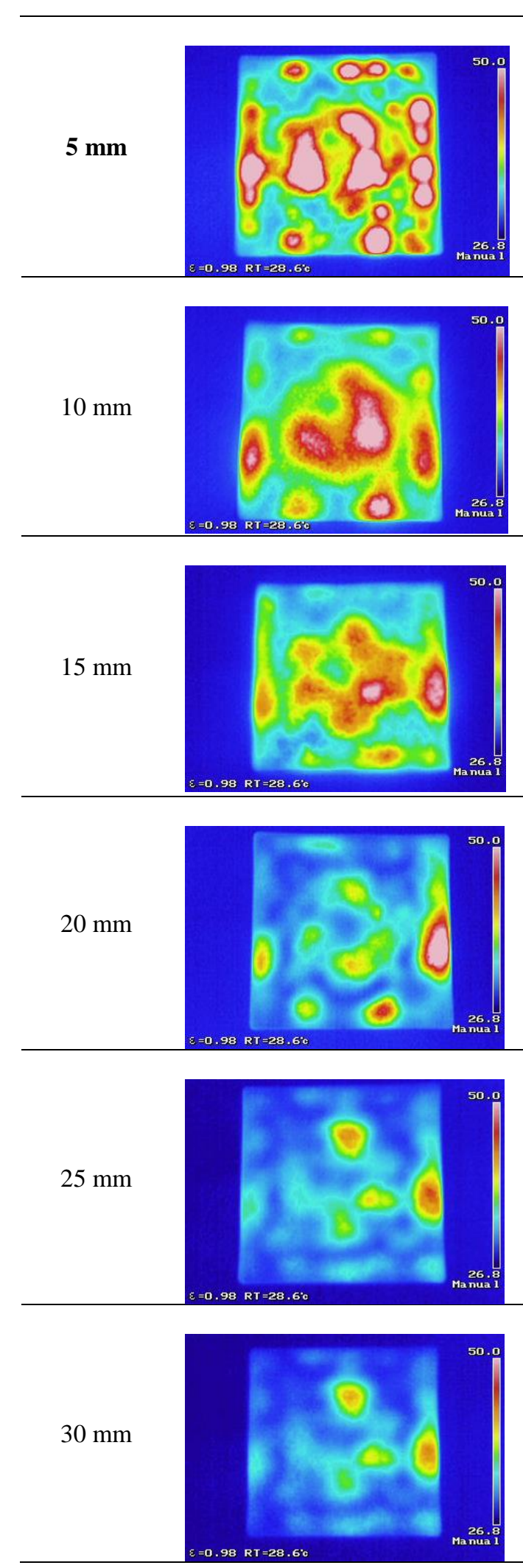
Heating Time

4 minutes 6 minutes
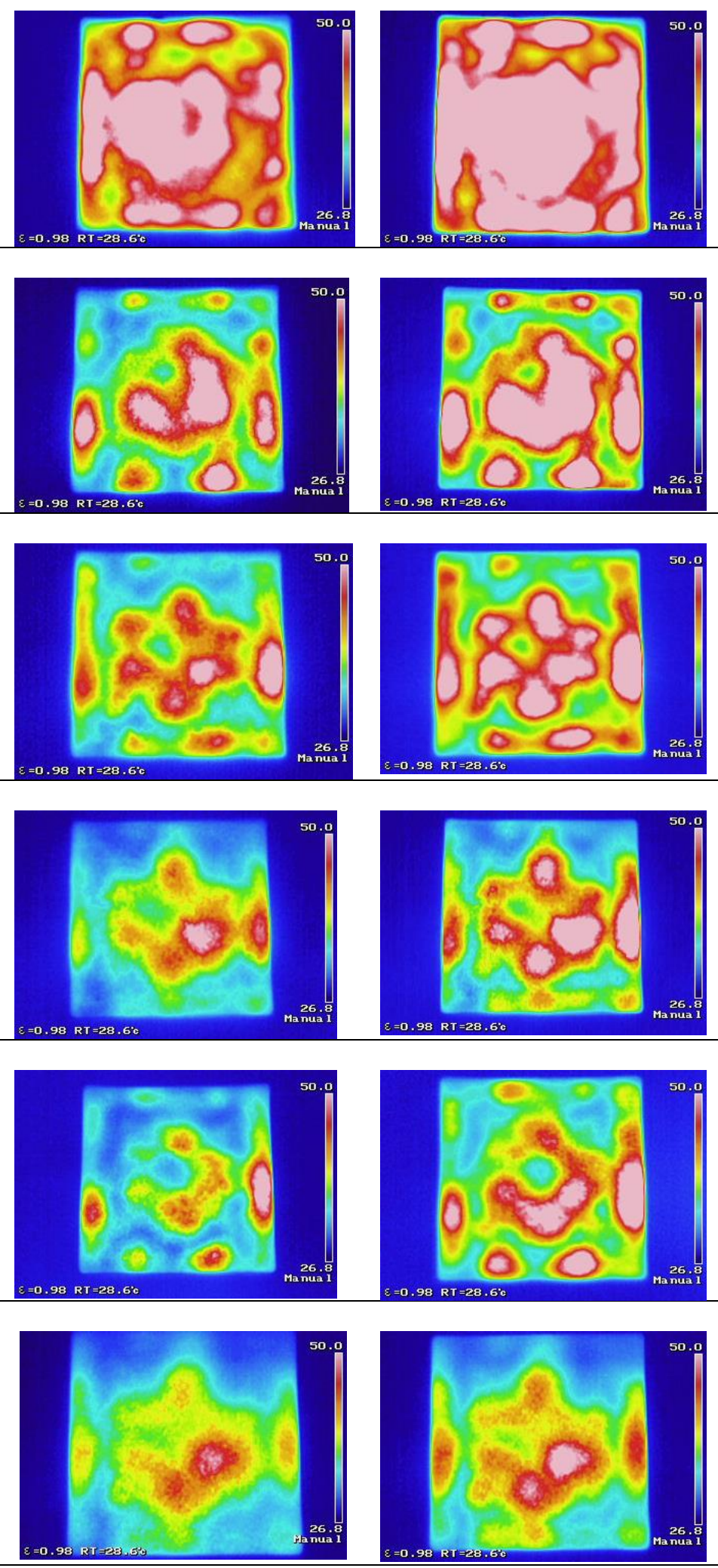


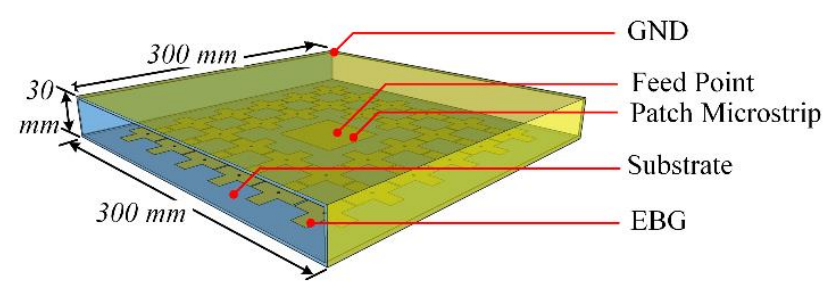

(a)

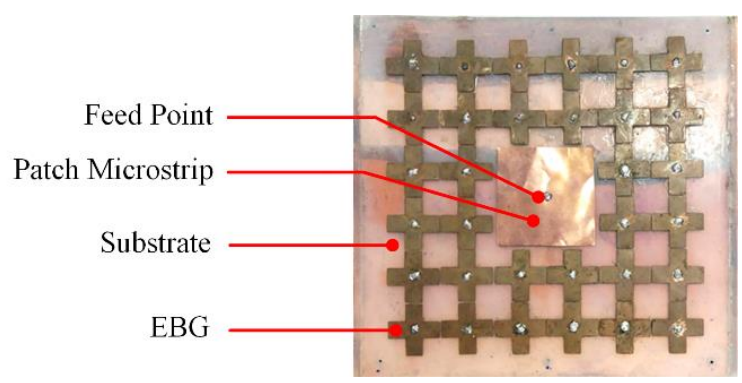

(b)

Figure. 12 Structure of: (a) a microwave dielectric heating chamber and (b) square patch microstrip together with plus-like EBG structure

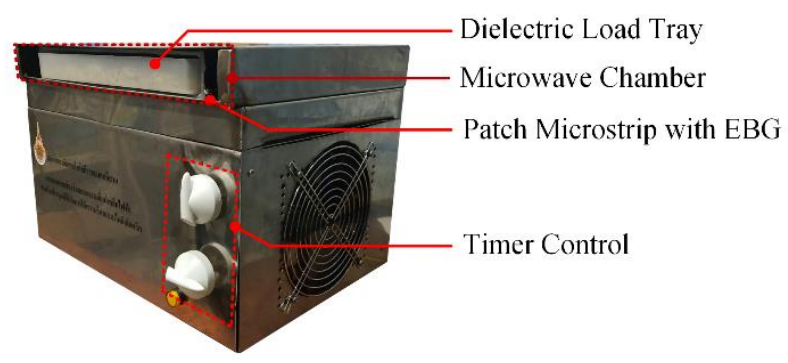

(a)

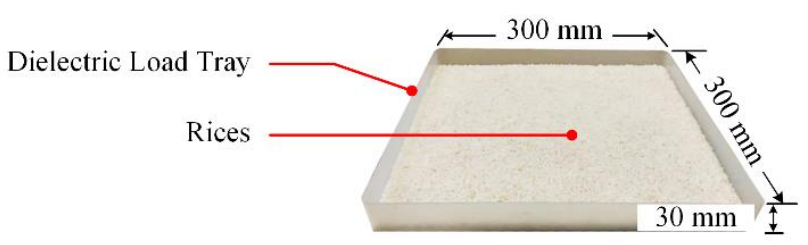

(b)

Figure. 13 Photograph of: (a) prototype of microwave dielectric heating machine and (b) polypropylene tray with dielectric load

directionality and continued to spread throughout the dielectric heating area. It can be seen that heating with a slight thickness dielectric load can make the dielectric load more evenly heated in a faster time. The electric field intensity in dielectric load was discussed in the simulation section because the electric field can influence the heat temperature, determined from Eq. (1). Considering to Eqs. (1) and (2), the greater the electric field intensity, the greater the load power density. In addition, the temperature was directly proportional to the power density and the time various also effects the temperature at dielectric load as illustrated in Eq. (2). From this relationship, it was shown that the simulation and experimental results were consistent.

Additionally, the researchers compare the efficiency of the proposed and traditional heating techniques. Traditionally technique, a magnetron tube was used to generate microwave power through a horn antenna as the research mentioned above $[3,8$, 10-14, 16, 19]. A microwave oven (Electrolux, model EMS 3067X, Stockholm, Sweden) was applied to test a traditional performance. The generated power, dielectric load thickness and weight were determined as 100 watts, $5 \mathrm{~mm}$ and $450 \mathrm{~g}$, respectively. The microwave oven works until the dielectric load temperature as $50{ }^{\circ} \mathrm{C}$ and takes 10 minutes to heat while the proposed technique takes only 6 minutes. This experimental research demonstrates that the use of microwave dielectric heating by using EBG together with patch microstrip emitter can save energy more than traditional one as 40 percentages.

\section{Conclusions}

The electromagnetic band gap (EBG) has appealed to be applied in microwave dielectric heating because of their desirable electric field properties. In this paper, increasing efficiency of applicator was designed and the electric field distribution at frequency of $2.45 \mathrm{GHz}$ over the applicator surface analyzed. It was found that the electric field was noticeably more distributed compared to the one port of plate applicator. The computation results of the electric field distribution on dielectric load as rice with the dielectric constant of 4.7, showed that the maximum electric field intensity was propagated thoroughly load under test with percentage of electric field distribution of $100 \%$ and $99.97 \%$ at top layer and middle layer, respectively, when dielectric thickness was $5 \mathrm{~mm}$. In this case study, a prototype of microwave heating chamber was constructed with one port microstrip applicator with plus shaped EBG. It was found that the temperature distributed measurement appeared to increase when the heating time was increased for the thickness of dielectric load.

In the future, by getting inspired by [32], we have a plan to design a proposed microwave applicator with more than one port. In addition, the designed microwave drying system will be analyzed for a commercial application of dielectric industrial drying.

\section{Conflicts of Interest}

The authors declare no conflict of interest. 


\section{Author Contributions}

The authors contribution as follow: Conceptualization, methodology, software, writing - original draft preparation, Nuchanart Santalunai and Samran Santalunai; validation, formal analysis, data curation, writing - review and editing, project administration, Nuchanart Santalunai, Samran Santalunai, Piyaporn Meesawad and Chanchai Tongsopa; Investigation, Experimental test, resources, visualization, Nuchanart Santalunai, Samran Santalunai and Samroeng Santalunai; supervision, Piyaporn Meesawad and Chanchai Tongsopa;

\section{Acknowledgments}

Thank you to the department of Telecommunications Engineering, Faculty of Engineering and Architecture, Rajamangala University of Technology Isan for providing support with the equipment. In addition, thank you to School of Electronic Engineering and Telecommunications Engineering, Institute of Engineering, Suranaree University of Technology for supporting this work with the equipment and the CST software.

Funding Statement: This work is supported by the SUT research and development fund.

\section{References}

[1] J. Xu, B. Wang, and Y. Wang, "Electromagnetic fields assisted blanching-Effect on the dielectric and physicochemical properties of cabbage", Journal of Food Process Engineering, Vol. 42, No. 8, 2019.

[2] Z. Mingu, O. Yebao, Z. Li, G. Tong, and W. Yunyang, "Effect of dielectric properties on radio frequency heating uniformity of apple", Nongye Gongcheng Xuebao/Transactions of the Chinese Society of Agricultural Engineering, Vol. 35, No. 20, pp. 273-279, 2019.

[3] M. Daian, A. Taube, G. Torgovnikov, G. Daian, and Y. Shramkov, "Computer Modelling of the Energy Distribution within Wood throughout Microwave Processing", CMC-Computers, Materials \& Continua, Vol. 8, No. 3, pp. 165172, 2008.

[4] R. Lamloumi, L. Hassini, G. L. Lecomte-Nana, M. A. Elcafsi, and D. Smith, "Modeling of Hydro-Viscoelastic State of Deformable and Saturated Product During Convective Drying", CMC-Computers, Materials \& Continua, Vol. 43, No. 3, pp. 137-152, 2014.
[5] J. A. Pandith and A. K. Srivastava, "Induction heating assisted foam mat drying of papaya pulp: Drying kinetics, drying modeling, and effects on quality attributes", Agricultural Engineering International: CIGR Journal, Vol. 20, No. 2, pp. 206-215, 2018.

[6] R. Maharjan, H. Yi, J. Ahn, G. H. Roh, C. Park, Y. Yoon, Y. Jang, I. Baek, Y. Kim, and S. Bae, "Effects of radiofrequency on the development and performance of Callosobruchus chinensis (Coleoptera: Chrysomelidae: Bruchinae) on three different leguminous seeds", Applied Entomology and Zoology, Vol. 54, No. 3, pp. 255-266, 2019.

[7] L. Zhang, R. Lan, B. Zhang, F. Erdogdu, and S. Wang, "A comprehensive review on recent developments of radio frequency treatment for pasteurizing agricultural products", Critical Reviews in Food Science and Nutrition, Vol. 61, No. 3, pp. 380-394, 2020.

[8] V. V. Komarov, Dielectric and Thermal Properties of Materials at Microwave Frequencies, Handbook, Artech House, A. H. 2012.

[9] H. P. van der Walt and D. V. Nicolae, "Modelling and Analysis of a Radio Frequency Dielectric Heating Method for Grinde Food Products", In: International Symposium on Power Electronics, Electrical Drives, Automation and Motion, Amalfi, Italy, pp. 10491054, 2018.

[10] G. Luo, C. Song, P. Hongjie, Z. Li, W. Xu, G. S. V. Raghavan, H. Chen, and G. Jin, "Optimization of the microwave drying process for potato chips based on the measurement of dielectric properties", Drying Technology, Vol. 37, No. 11, pp. 1329-1339, 2019.

[11] J. Zhang, M. Li, J. Cheng, J. Wang, Z. Ding, X. Yuan, S. Zhou, and X. Liu, "Effects of moisture, temperature, and salt content on the dielectric properties of pecan kernels during microwave and radio frequency drying processes", Foods, Vol. 8, No. 9, pp. 1-14, 2019.

[12] C. Singh, N. Saluja, and R.K. Sharma, "A computation-driven, energy-efficient and hybrid of microwave and conventional drying process for fast gooseberry candy production", Journal of Microwave Power and Electromagnetic Energy, Vol. 53, No. 4, pp. 259-275, 2019.

[13] Y. Llave, D. Kambayashi, M. Fukuoka, and N. Sakai, "Power absorption analysis of twocomponent materials during microwave thawing and heating: Experimental and computer simulation", Innovative Food Science and 
Emerging Technologies, Vol. 66, No. 102479, pp. 1-14, 2020.

[14] M. A. Fouad, M. Gaber, K. Knoerzer, M. P. Mansour, F. J. Trujillo, P. Juliano, and P. Shrestha, "Improved canola oil expeller extraction using a pilot-scale continuous flow microwave system for pre-treatment of seeds and flaked seeds", Journal of Food Engineering, Vol. 284, No. 110053, pp. 1-11, 2020.

[15] X. Zhou and S. Wang, "Recent developments in radio frequency drying of food and agricultural products: A review", Drying Technology, Vol. 37, No. 3, pp.271-286, 2019.

[16] J. Zhang, M. Li, Z. Ding, J. Cheng, S. Yang, and $X$. Liu, "Microwave airflow drying of pecans at variable microwave power", Journal of Food Process Engineering, Vol. 42, No. 1, 2019.

[17] B. Ling, L. Hou, R. Li, and S. Wang, "Storage stability of pistachios as influenced by radio frequency treatments for postharvest disinfestations", Innovative Food Science and Emerging Technologies, Vol. 33, No. 1, pp. 357364, 2016.

[18] L. Zhou, B. Ling, A. Zheng, B. Zhang, and S. Wang, "Developing radio frequency technology for postharvest insect control in milled rice", Journal of Stored Products Research, Vol. 62, No. 1, pp. 22-31, 2015.

[19] B. Ling, G. Tiwari, and S. Wang, "Pest control by microwave and radio frequency energy: dielectric properties of stone fruit", Agronomy for Sustainable Development, Vol. 35, No. 1, pp. 233-240, 2015.

[20] M. Mehdizadeh, Microwave/RF Applicators and Probes for Material Heating, Sensing, and Plasma Generation: A Design Guide, Oxford, UK, William Andrew, W.A.2015.

[21] D. R. Sridhar, "Periodic EBG structure based UWB band pass Filter", International Journal of Advanced Research in Electrical, Electronic and Instrumentation Engineering, Vol. 2, No. 5, pp. 1682-1686, 2013.

[22] N. Kushwaha and R. Kumar, "Study of different shape Electromagnetic Band Gap (EBG) structures for single and dual band applications", Journal of Microwaves, Optoelectronics and Electromagnetic Applications, Vol. 13, No. 1, pp. 16-30, 2014.

[23] H. F. Shaban, H. A. Elmikatyn and A. A. Shaalan, "Study the effects of electromagnetic band-gap (EBG) substrate on two patches microstrip antenna", Progress, In Electromagnetics Research B, Vol. 10, No. 1, pp. 55-74, 2008.
[24] F. Yang and Y. Rahmat-Samii, Electromagnetic Band Gap Structure in Antenna Engineering, Cambridge University Press, New York, N.Y.2009.

[25] N. Fhafhiem, P. Krachodnok, and R. Wongsan, "Curved strip dipole antenna on EBG reflector plane for RFID applications", WSEAS Transactions on Communications, Vol. 9, No. 6, pp. 374-383, 2010.

[26] L. Li, B. Li, H. Liu, and C. Liang, "Locally resonant cavity cell model for electromagnetic band gap structures", IEEE Transactions on Antennas and Ppropagation, Vol. 54, No. 1, pp. 90-100, 2006.

[27] L. Yang, M. Fan, F. Chen, J. She, and Z. Feng, "A novel compact electromagnetic-bandgap (EBG) structure and its applications for microwave circuits", IEEE Transactions on Microwave Theory and Techniques, Vol. 53, No. 1, pp. 183-190, 2005.

[28] C. A. Balanis, Antenna Theory, Hoboken Press, 3rd ed., New Jersey, N.J.2004.

[29] S. Santalunai, T. Thosdeekoraphat, and C. Thongsopa, "Optimization of Electric Field Distribution on Capacitor Copper Plate by Increasing Input Power Ports for Dielectric Heating Applications", Australian Journal of Basic and Applied Sciences, Vol. 8, No. 7, pp. 58-65. 2014.

[30] S. Santalunai, T. Thosdeekoraphat and C. Thongsopa, "The Effect of Electric Field Distributions on Grains and Insect for Dielectric Heating Applications. International Journal of Biological", Veterinary, Agricultural and Food Engineering, Vol. 8, No. 7, pp. 667-673. 2014.

[31] B. D. Davidson, Computational Electromagnetics for $R F$ and Microwave Engineering, Cambridge University Press, New York, N.Y.2011.

[32] S. Santalunai, W. Charoensiri, S. Santalunai, T. Thosdeekoraphat, C. Thongsopa, N. Fhafhiem, "Electric Fields Distribution of Optimized Waveguide Potrs Power Position for Grains Drying Applications", In: Proc. of International Video Conf. on Science Technology and Management, Paris, France, pp. 31-35, 2020. 